\title{
Minimal access left ventricular reconstruction
}

\author{
Thasee Pillay $^{1}$, Paulo Neves ${ }^{2}$, federico benetti ${ }^{3}$, Kevin van Bladel ${ }^{1}$, Andrew Wechsler ${ }^{1}$, and \\ Lon Annest ${ }^{1}$ \\ ${ }^{1}$ BioVentrix Inc \\ ${ }^{2}$ Centro Hospitalar de Vila Nova de Gaia Espinho EPE \\ ${ }^{3}$ Affiliation not available
}

July 6, 2020

\begin{abstract}
The Revivent TC TransCatheter Ventricular Enhancement System (BioVentrix Inc, San Ramon, CA, USA) is intended for use in heart failure with cardiac dysfunction a previous myocardial infarction. The resultant increased left ventricular systolic volume and discrete, contiguous, non-contractile (akinetic and/or dyskinetic) scar located in the antero-septal, apical (may extend laterally) region of the left ventricle (LV) lends itself to Revivent. The procedure, called Less Invasive Ventricular Enhancement (LIVE), consists of the implantation of a series of micro-anchors pairs in order to exclude the scarred myocardium, in order to reduce and reshape the LV. We present the procedure step-by-step, as team coordination between the cardiac surgeon and the interventional cardiologist is essential to ensure good procedural outcomes. This is a novel and new technique to address Heart Failure secondary to Myocardial Infarction.
\end{abstract}

\section{Introduction}

At present, over 15 million people are living with heart failure in Europe. Heart failure is responsible in most westernized economies for 1-2\% of all healthcare expenditures and is the leading cause of hospitalization in people over the age of 65 . The prognosis for HF patients has remained alarmingly poor over the last twenty years. Despite some notable improvements in survival rates, approximately 1 in 3 patients admitted to a hospital with HF still die within one year. This places a huge financial burden on the health care system and signals the typical downward spiral of the course of this disease. Due to this fact, there has been a significant paradigm shift in the focus of cardiac treatment worldwide in last two decades.

It has been common knowledge since the pivotal report by White et al. that left ventricular (LV) chamber size is the single most important metric in determining survival post myocardial infarction (MI). Ischemic cardiomyopathy is a disease process wherein the patient develops HF due to scar formation and subsequent LV enlargement. Ischemic cardiomyopathy is the largest cause of HF, comprising approximately two-thirds of all the 22 million HF patients worldwide. Analysis of the 10-year survival of these patients based on LV size demonstrates the rapid divergence in death rate in the initial several years. Hence, developing a safe and effective approach for volume reduction in this most vulnerable patient segment is the significant challenge in modern cardiac care.

Ischemic cardiomyopathy is frequently associated with LV scarring where viable heart tissue has been damaged and is replaced by fibrotic scar, which results in left ventricular dysfunction. The loss in left ventricular function most often results in angina, significant dyspnoea, ventricular arrhythmias, and an increased risk of thromboembolism and death.

Surgical treatment is an option in advanced HF. Patients with significantly enlarged LV end-systolic and enddiastolic volume indices (LVESVI and LVEDVI) appear to benefit most from surgical intervention. Relative 
contraindications to surgery include excessive anaesthetic risk, impaired function of residual myocardium, severely diminished cardiac index, and lack of a discrete scar with indistinct margins. Left ventricular surgical treatment usually requires a median sternotomy, cardiopulmonary bypass, and left ventriculotomy on either a beating or arrested heart. Adoption of the surgical technique for correction of this condition has been limited by both the invasiveness of the procedure and potential for significant subsequent perioperative morbidity and mortality. BioVentrix has developed the Revivent TC System to provide devices for the treatment of a left ventricular scar, utilizing a less invasive, beating heart approach.

Less Invasive Ventricular Enhancement (LIVE) procedure with Revivent TC reshapes and resizes LV without sternotomy or extracorporeal circulation through direct plication of the scarred myocardium. The procedure consists in the implantation of a series of internal (right ventricular septum) and external titanium microanchors $(5 \mathrm{~mm} \times 25 \mathrm{~mm})$ which are brought together through a poly-ether-ether-ketone (PEEK) tether $(1.7$ $\mathrm{mm} \times 1.0 \mathrm{~mm}$ ) to approximate LV free wall and the anterior septum, consequently excluding the scarred myocardium. Septal RV anchors are deployed by a transcatheter technique, through a venous access on the neck - right internal jugular vein.

First-in-human LIVE procedures were performed in September 2013. Since the device obtained CE mark approval in June 2016, the multicentric observational BioVentrix Registry Assessment of Ventricular Enhancement for the Revivent TC system (BRAVE-TC) trial has been ongoing in Europe (1).

In total, 230 cases have been performed with an overall mortality of 18 (7.8\%). 12 cases of mortality occurred during the CE mark trial and 4 prior to changing the procedure to apical snaring (described below).

One-year results of a multicentric study were recently published by Klein et al. (2), including 86 patients treated with Revivent TC system has registered a significant improvement in LV ejection fraction (29 +$8 \%$ vs $34+-9 \%, \mathrm{p}<0.005)$ and an important reduction of LV volumes - LV end-systolic and end-diastolic volume indexes (LVESVi and LVEDVi) both decreased: $74+-28 \mathrm{~mL} / \mathrm{m}^{2}$ vs $54+-23 \mathrm{~mL} / \mathrm{m}^{2}, \mathrm{p}<0.001$ and $106+-33 \mathrm{~mL} / \mathrm{m}^{2}$ vs $80+-26 \mathrm{~mL} / \mathrm{m}^{2}$, respectively, $\mathrm{p}<0.0001$. Mortality was $4.5 \%$ (4 patients) and 12 -months survival was $90.6 \%$. There was also a documented clinical improvement of the treated patients. This is the largest series of patients submitted to LIVE therapy published so far.

In another series of 26 patients submitted to the LIVE therapy (3), meaningful LV reduction was achieved - LVSEVi went from 84.8 +- 25.7 to 65.6 +- $24.4 \mathrm{ml} / \mathrm{m}^{2}$ and LVEDVi went from 107.8 +- 33.2 to 90.5 +$31.8 \mathrm{ml} / \mathrm{m}^{2}$, both $\mathrm{p}<0.001$ (Figure 1 ). Additionally, ejection fraction improvement was observed by CMR: $28.9+-8.3 \%$ vs $38.6+-10.5 \%, \mathrm{p}<0.001$. There was one mortality case reported. Lastly, also the 6 -minute walk test was significantly longer $(368.8+-40.0$ to $461.5+-61.2 \mathrm{~m}, \mathrm{p}<0.001)$, endorsing clinical benefit with the technique.

Between July 2018 and January 2020, 47 patients (84.4\% men; mean age $61+-12.5$ years) were submitted to the LIVE procedure in 16 Institutions in Europe, North America and Asia. Procedural success was 100\%. There was no intra-hospital mortality. There was no case of ventricular septal defect, right ventricular perforation or sternotomy conversion. New onset tricuspid valve regurgitation was observed in one patient. In the mean follow-up period of 9.8 months, NYHA class improved a median of 1 grade and there was no late mortality.

We present this approach here, describing it step-by-step.

\section{Patient selection}

Any patients suffering from heart failure symptoms with cardiac dysfunction caused by a previous myocardial infarction resulting in increased LV systolic volume and in a discrete, contiguous, non-contractile, (akinetic and/or dyskinetic) scar located in the antero-septal, apical (may extend laterally) region of the left ventricle.

Transthoracic echocardiography (TTE) is utilized to assess for LV dilatation, diminished ejection fraction (EF), LVESVI and LVEDVI, regional wall akinesis or dyskinesis. It is a useful imaging modality to guide further investigation to determine suitability for Revivent TC and to plan the LV reconstruction. 
Cardiac magnetic resonance (CMR) with late gadolinium enhancement (LGE) gives a higher resolution image, clearly indicating scar thickness, extent and anatomy. It also gives excellent indication of LVESVI, LVEDVI and EF. LGE is the gold standard for scar determination.

$4 \mathrm{D}$ cardiac computed tomography $(\mathrm{CT})$ scan carried out with a triphasic injection of contrast media is a suitable alternative to CMR for patients unable to undergo the latter.

The following are absolute contra-indications for the LIVE procedure:

- Myocardial Infarction within 90 days prior to the procedure;

- Previous right neck surgery or previous left chest surgery that precludes device placement;

- Thrombus or intra-ventricular mass in the left atrium or ventricle as verified by echocardiography or equivalent that has not been adequately treated with weeks of anticoagulant at therapeutic levels;

- Calcified ventricular wall in the area of intended scar exclusion as verified by one or more appropriate imaging modalities.

The following are relative contra-indications for the LIVE procedure:

- Chronic renal failure with a serum creatinine $>2 \mathrm{mg} / \mathrm{dL}$;

- Inoperable coronary disease with significant ischemia;

- Inadequate myocardial viability in regions remote from the scar;

- Cardiac Resynchronization Therapy (CRT) consisting of bi-ventricular pacemaker device (i.e., not ICD only) placement [?] 60 days prior to treatment;

- Patient intolerance or unwillingness to take anti-coagulation medication;

- Functioning pacemaker leads in antero-apical RV, which, in the opinion of the investigator, would interfere with anchor placement;

- Pulmonary Arterial Pressure > $60 \mathrm{~mm} \mathrm{Hg}$ shown by right heart catheterization to be precapillary or unresponsive to vasodilator therapy;

\section{Equipment}

The implantable components consist of a series of titanium anchor pairs (one Internal and one External in each pair) covered in polyester cloth (Figure 1 ).

The anchor pairs are connected to one another by an adjustable-length tether made of poly-ether-ether ketone (PEEK). Distance between anchors is variable and is determined by the position of the External Anchor on the Tether. The Internal Anchor is hinged to facilitate low profile passage through a jugular vein catheter that is inserted perpendicularly to the septum, after which it can be pivoted 90deg to lie flat on the septum.

The External Anchor is delivered through mini-thoracotomy and houses a cam-based reversible locking mechanism allowing apposition of the two anchors at a continuum of positions. Once the proper distance and appropriate Tether force between the two is established, excess Tether length is cut and removed.

The Revivent TC System consists of:

- Internal Anchor Flex Assembly

- External Anchor Assembly

- Curved Needle, LV Apex Anchor

- Introducer Kit

- Trans Thoracic Force Gauge

\section{Implant procedure}

The patient shall be prepared for cardiac hybrid procedure according to hospital's standard procedures. Intraoperative transoesophageal echocardiography (TEE) is required to provide procedural assistance, monitor LV and valve function, and evaluate myocardial anchor implant placement. 
A cell salvage system, standby cardiopulmonary bypass, external defibrillator pads, meticulous control of $\mathrm{ACT}$ and serum $\mathrm{K}^{+}$and $\mathrm{Mg}^{2+}$ add to patient safety.

We are describing the procedure step-by-step:

1. Percutaneously access the right internal jugular (RIJ) vein.

2. Insert a $14 \mathrm{~F}$ Introducer into RIJ and advance into the superior vena cava (SVC) or right atrium (RA) (Figure 3).

3. Determine location of incision by placing an instrument under fluoroscopy at the desired location and ensure that the position allows access to the LV apex and placement of the most basal anchor pair. The incision should be placed over the intercostal space that gives a direct line from the antero-lateral wall 5-6 cm above the apex of the $\mathrm{LV}$ and $1-2 \mathrm{~cm}$ above the very apex of the RV; in general, the medial aspect of the incision should be at the level of the patient's areola. This imaging should be repeated before actually entering the interspace.

4. Perform the thoracotomy.

5. Remove extra-pericardial fat and open the pericardium under direct vision.

6. Identify aneurysm (scar) margins and epicardial landmarks including LAD and cardiac apex.

7. Place a "leash" or tethering mechanism to manipulate the heart. The "leash" consists of a 2-0 double pledgeted, horizontal mattress polypropylene suture with a tourniquet. Suture should be placed in the scar tissue of the heart using suture and pledgets appropriately deep in the scar to prevent pulling through the wall of the heart. The site of the leash should be about half way between the lateral scar margin and the LAD at the level of the RV-apical anchor on the LV epicardium. Additional leashes may be placed in the scar as necessary to allow appropriate cardiac manipulation. Leashes should be placed only in scar intended for exclusion.

8. Heparinize the patient maintaining an ACT of 300 seconds or greater. Float a Swan-Ganz catheter through the 14 Fr sheath up to the pulmonary artery (PA).

9. Advance a 0.025 Jagwire through the Swan-Ganz catheter and keep it on the PA.

10. Advance a 7Fr multipurpose (MP) catheter with a EnSnare endovascular snare system inside and open it in the RV apex (Figure 4) .

11. Perform a RV gram to confirm the position of the EnSnare

12. Place a $7 \mathrm{~cm} 18$ gauge straight needle on the scarred epicardium to identify landmarks and needle puncture direction. (Caution: avoid placing needle within healthy myocardium).

13. Puncture the needle through the epicardial surface and redirect it towards the selected septal site as directed by the EnSare in the RV apex; (confirm LV wave form with pressure measurement). Confirm needle is in the RV using injection of contrast within the needle(Figure 5) .

14. Advance a 0.032" wire through the needle and snare it with the EnSnare under fluoroscopy.

15. At this point, the $14 \mathrm{Fr}$ sheath is advanced to the RV, near the interventricular septum. The 0.025" Jagwire can be also removed.

16. Remove the straight needle and advance a $6 \mathrm{Fr}$ Brite tip catheter through the 0.032 " wire and into the RV (confirm scar location). Tactile feedback should reinforce the catheter's passage through scar. This is done under fluoroscopy and TEE should be continuously looking at any changes in tricuspid regurgitation change from baseline(Figure 6) .

17. Exchange the 0.032 " wire with a 0.014 " through a $6 \mathrm{Fr}$ standard multipurpose catheter. This is done in the surgical side.

18. At this point, the $6 \mathrm{Fr}$ Brite tip catheter must be inside the $14 \mathrm{Fr}$ sheath.

19. Place the Internal Anchor assembly over the 0.014" guidewire, and embed the OWTI tip into the end of the guiding catheter; place a small clamp on both exposed ends of the 0.014" guidewire - one where it exits the OWTI on the jugular side of the patient, and the other where the guidewire exits the guiding catheter at its hub. (This maneuver creates a single entity out of the OWTI and the guiding catheter so they may be advanced from the jugular access on the patient's right side, to the epicardial access on the left side).

20. Advance the OWTI/guiding catheter combination through the $14 \mathrm{Fr}$, and then through the $6 \mathrm{Fr}$ Brite 
tip, which is tranversing two walls of the LV. During this maneuver, the $14 \mathrm{Fr}$ introducer must be kept in contact with the right side of the septum, with the tip of the $6 \mathrm{Fr}$ Brite tip inside the $14 \mathrm{Fr}$ lumen. The 0.014" guidewire and guiding catheter may be removed as soon as any part of the wire becomes exposed outside the 6Fr Brite tip at the epicardial location (Figure 7).

21. The tip of the $14 \mathrm{Fr}$ introducer, which has been essentially in contact with the septum, is retracted to reveal the anchor when the OWTI has been advanced to bring the anchor into immediate proximity of the septum.

22. Carefully lay down the hinged, retrievable internal anchor onto the septum, such that the hinged surface is lying in position on the septum. Gentle tension must be maintained continuously on the tether from the LV side. With the hinged anchor laying on the septum, the OWTI is cut in the rectangular portion of the tether (leaving as much of the tether length as possible); remove the 6Fr Brite tip. The external anchor is placed over the tether and advanced to the epicardial surface. (Bleeding may occur briefly after removal of the $6 \mathrm{Fr}$ sheath; contact between the external anchor and the epicardium generally creates hemostasis). Then use the force gauge to bring the two walls of the LV into apposition (Figure 8).

23. Assess hemodynamics, LV configuration (by TEE), and tricuspid valvular function (by TEE); if appropriate, release anchor from Flex Catheter, leaving it in position against the right side of the septum.

24. Retract the $14 \mathrm{Fr}$ sheath at least to within the RA proximal to the tricuspid annulus, but ideally to the cavo-atrial junction immediately after successful deployment and release of the hinged, retrievable internal anchor onto the septum.

25. If there are no more RV anchors anticipated, leave the walls in contact-plus-1 (one) Newton (using the force gauge). If more are planned, release the external anchor to allow separation between the endocardial surfaces to avoid distortion of the LV chamber for subsequent anchor placement.

26. Repeat steps 13-22 for each LV-RV anchor pair.

27. When it is determined that the next anchor should be LV-LV (i.e., at the LV apex with no further space in the RV apex), retract the introducer from the RV, then oppose the walls with existing anchor pairs into contact plus 1-2 Newtons, (thus rendering the geometry of the final, reshaped LV except for the apex). Caution: Using excessive force could lead to tissue erosion or anchor migration, it is critical to use the force gauge to ensure proper force to the anchors is being applied.

28. Using the LV Apical needle in a standard surgical needle holder, load the needle such that it is at a 10 to 15-degree angle from the needle holder shaft.

29. With visibility gained from manipulation of previous anchor pairs, drive the LV needle across the cardiac apex from right-to-left.

30. Retrieve the needle tip. Lay down the hinged anchor on the right side of the apex and cut the tether at or near the needle to allow placement of an external anchor for hemostasis. Check all anchor positions fluoroscopically for alignment (Figure 9).

\section{Discussion}

Both Revivent TC approaches are essentially less invasive than conventional SVR. Therapeutic volume reduction was achieved regardless of delivery method. Interestingly, there was no additional benefit of adding coronary revascularization to the procedure. This data compares favourably with the STICH sub-analysis, which established a survival benefit in patients realizing $>30 \%$ reduction in LVESVI and/or post-operative LVESVI $<60 \mathrm{ml} / \mathrm{m}^{2}(4)$.

Surgical ventricular reconstruction or SVR, has been applied clinically in a large number of patients during the past two decades (5-9).In the STS database, for a 10 year period ending 2017, 2273 SVR procedures were undertaken. Mortality ranged between 8 and 15\%. During the same period, $7205 \mathrm{LV}$ aneurysm repairs were carried out.

SVR improves heart failure symptoms and long-term survival for patients with ischemic cardiomyopathy (10). The majority of cases in these studies underwent standard open-heart surgery via sternotomy with cardiopulmonary bypass, cardioplegic myocardial arrest, and ventriculotomy. Concomitant coronary revas- 
cularization was performed in most cases, sometimes also in combination with an intervention to the mitral valve for functional or secondary mitral valve regurgitation. Implantation of the Revivent TC System device does not require cardiopulmonary bypass, cardioplegic arrest, or a ventriculotomy. Implantation was initially performed with sternotomy, followed by the hybrid approach. Both approaches are less-invasive compared to standard SVR procedures.

The outcomes and the rate of adverse events during and after implantation of the Revivent TC System appear to be in an acceptable range when compared with SVR. The 30 day mortality of $5 \%$ in this group of patients is within the range of STS mortality. Hospital stay could be significantly reduced by using the hybrid approach rather than the initial surgical approach. The observed 1- and 2-year survival of $91 \%$ and $89 \%$, respectively, are also comparable to SVR outcomes $(11,12,13,14)$.

By comparison, the reported survival from the international Reconstructive Endoventricular Surgery returning Torsion Original Radius Elliptical shape to the left ventricle (RESTORE) registry of 1,198 post-anterior infarction SVR cases at 2-years was $~ 85 \%$. Improvement in outcomes after implantation of the Revivent TC System should be possible through application of experience gained in selecting candidates and in the technique of implantation. This might also offer an alternative in patients at high risk of perioperative complications or with a frail preoperative condition.

An important element of the Revivent TC System implantation technique is that the anchor pairs are set to a configuration parallel to the long axis of the heart. Each tether and the excluded portion of the scar is taken from the short axis of the heart. With this configuration, virtually all volume reduction decreases the radius of the $\mathrm{LV}$ and is not just the result of amputation of an apical aneurysm. Aligning good functioning myocardium edge to edge optimizes myocardial function. This is not the case with a Dacron patch which only reduces size of the LV cavity but offers an akinetic neo LV wall. Reduction in wall tension, reorientation of myofibers, and improvement in torsional dynamics is, therefore, the most likely explanation for the functional improvement observed in the patients.

\section{Conclusion}

With Revivent Transcatheter, Left Ventricular Reconstruction is highly feasible, safe and efficacious. The avoidance of median sternotomy, cardiopulmonary bypass and cross clamping with cardioplegia allows for immediate haemodynamic improvement and shorter recovery and return to normal activities. The volume reduction is comparable to most surgical series and fares much better than the STICH values. Ongoing studies will further demonstrate the value of this approach in this sick group of heart failure patients.

\section{Figure legends}

Figure 1 - Pre and post-procedural cardiac magnetic resonance, showing LV volume reduction and reshape achieved with Revivent TC system.

Figure 2 - The Revivent TC System consists of titanium anchors and delivery instrumentation for the anchor placement.

Figure 3 - step 2 - Insert a 14F Introducer into RIJ and advance into the superior vena cava (SVC) or right atrium $(\mathrm{RA})$.

Figure 4 - step 10 - Advance a 7Fr multipurpose (MP) catheter with a EnSnare endovascular snare system inside and open it in the RV apex.

Figure 5 - step 13 - Puncture the needle through the epicardial surface and redirect it towards the selected septal site as directed by the EnSare in the RV apex.

Figure 6 - step 16 - Remove the straight needle and advance a 6Fr Brite tip catheter through the 0.032" wire and into the RV (confirm scar location).

Figure 7 - step 20 - Advance the OWTI/guiding catheter combination through the $14 \mathrm{Fr}$, and then through the $6 \mathrm{Fr}$ Brite tip, which is tranversing two walls of the LV. 
Figure 8 - step 22 - The external anchor is placed over the tether and advanced to the epicardial surface. Then use the force gauge to bring the two walls of the LV into apposition.

Figure $\mathbf{9}$ - final step - final LV gram assessment and pre-operative comparison

Author contributions

TP and PN did article drafting and revision, FB did critical revision of the article, KvB, AW and LA were involved in device development and proctoring the majority of the procedures.

\section{References}

1. Observational study to collect data on health improvements after mini-invasive operation with Revivent TC device to reshape the heart and reduce its volume: BioVentrix Registry Assessment of Ventricular Enhancement for the Revivent TC system (BRAVE- TC). https://doi.org/10.1186/ISRCTN89757315, 2016; URL http://www.isrctn.com/ISRCTN89757315.

2. Klein P, Anker SD, Wechsler A. Less invasive ventricular reconstruction for ischaemic heart failure. Eur J Heart Fail. 2019 Dec;21(12):1638-1650.

3. Wang Y, Xiao G, Zhang G, et al. Early Results of the Revivent TC Procedure for Treatment of Left Ventricular Aneurysm and Heart Failure due to Ischemic Cardiomyopathy. EuroIntervention. 2020 Jan 28. pii: EIJ-D-19-00225

4. Rouleau JL, Michler RE, Velazquez EJ, et al. The STICH trial: evidence-based conclusions. Eur J Heart Fail. 2010;12(10):1028- 1030.

5. Reddy et al Left ventricular aneurysm: Twenty-year surgical experience with 1572 patients at the Texas Heart Institute. Cardiovasc Dis. 1981 Jun;8(2):165-186.

6. Guilmet D, Popoff G, Dubois C, Tawil N, Bachet J, Goudot B, Guermonprez JL, Brodaty D, Schlumberger S A new surgical technic for the treatment of left ventricular aneurysm: the overcoat aneurysmoplasty. Preliminary results. 11 cases. Arch Mal Coeur Vaiss. 1984 Aug;77(8):953-8. French

7. Jatene AD Left ventricular aneurysmectomy. Resection or reconstruction. J Thorac Cardiovasc Surg. 1985 Mar;89(3):321-31. No abstract available.

8. Dor V, Saab M, Coste P, Kornaszewska M, Montiglio F. Left ventricular aneurysm: a new surgical approach. Thorac Cardiovasc Surg. 1989 Feb;37(1):11-9.

9. Benetti $\mathrm{FJ}^{1}$,et al. A new surgical technique for fibrosed interventricular septum J Cardiovasc Surg (Torino). 1990 Jan-Feb;31(1):36-40

10. Calafiore $\mathrm{AM}^{1}$, Iaco AL, Amata D, Castello C, Varone E, Falconieri F, Bivona A, Gallina S, Di Mauro M. Left ventricular surgical restoration for anteroseptal scars; volume versus shape

11. Buckberg GD. Ventricular restoration - a surgical approach to reverse ventricular remodeling. Heart Fail Rev 2004;9: 233-239.

12. Jones RH, Velazquez EJ, Michler RE, et al. Coronary bypass surgery with or without surgical ventricular reconstruction. N Engl J Med. 2009 Apr 23;360(17):1705-17.

13. Hassanabad AF, MacQueen KT, Ali I. Surgical Treatment for Ischemic Heart Failure (STICH) trial: A review of outcomes. J Card Surg. 2019 Oct;34(10):1075-1082.

14. Isomura T, Hoshino J, Fukada Y, et al. Volume reduction rate by surgical ventricular restoration determines late outcome in ischaemic cardiomyopathy. Eur J Heart Fail. 2011;13(4):423-431. 


\section{LIVE with Revivent TC ${ }^{\mathrm{TM}}$}

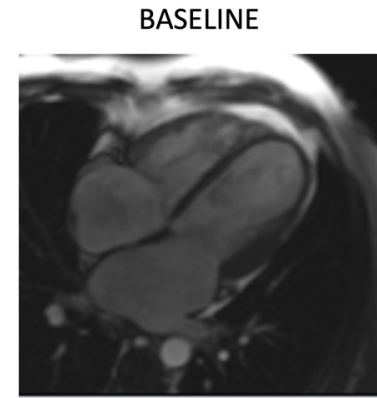

LVESVI $=127 \mathrm{~mL} / \mathrm{m}^{2}$
POST-Op (6M)

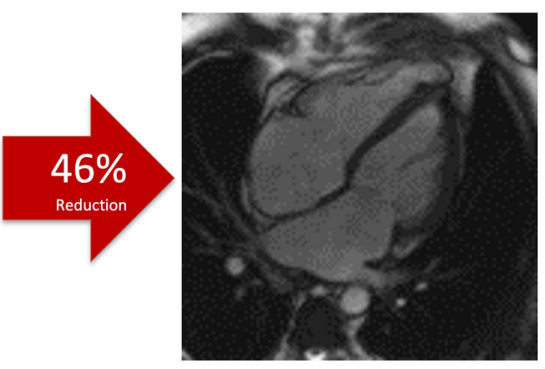

LVESVI $=69 \mathrm{~mL} / \mathrm{m}^{2}$

8 BioVentrix

Less Invasive Ventricular Enhancement

REVIVENT TC MECHANISM OF ACTION

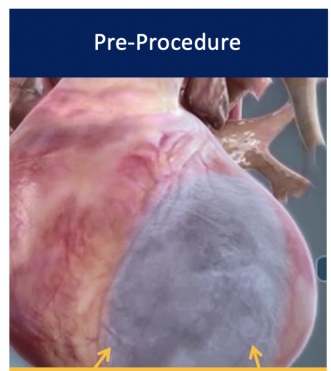

Dilated cardiomyopathy due to post-MI scarring

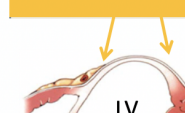

LV
Implant Revivent TC Anchors

Revivent TC Anchors

and Tether

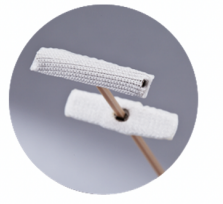

Instrumentation for tether

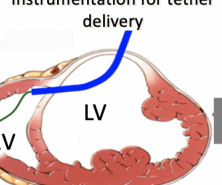

RV
Post-Procedure

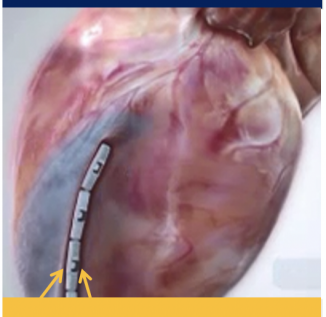

Scar excluded, LV wall tension decreased and function improved

Internal Anchor External

$\mathrm{RV}$
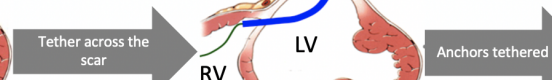

$R$

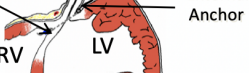



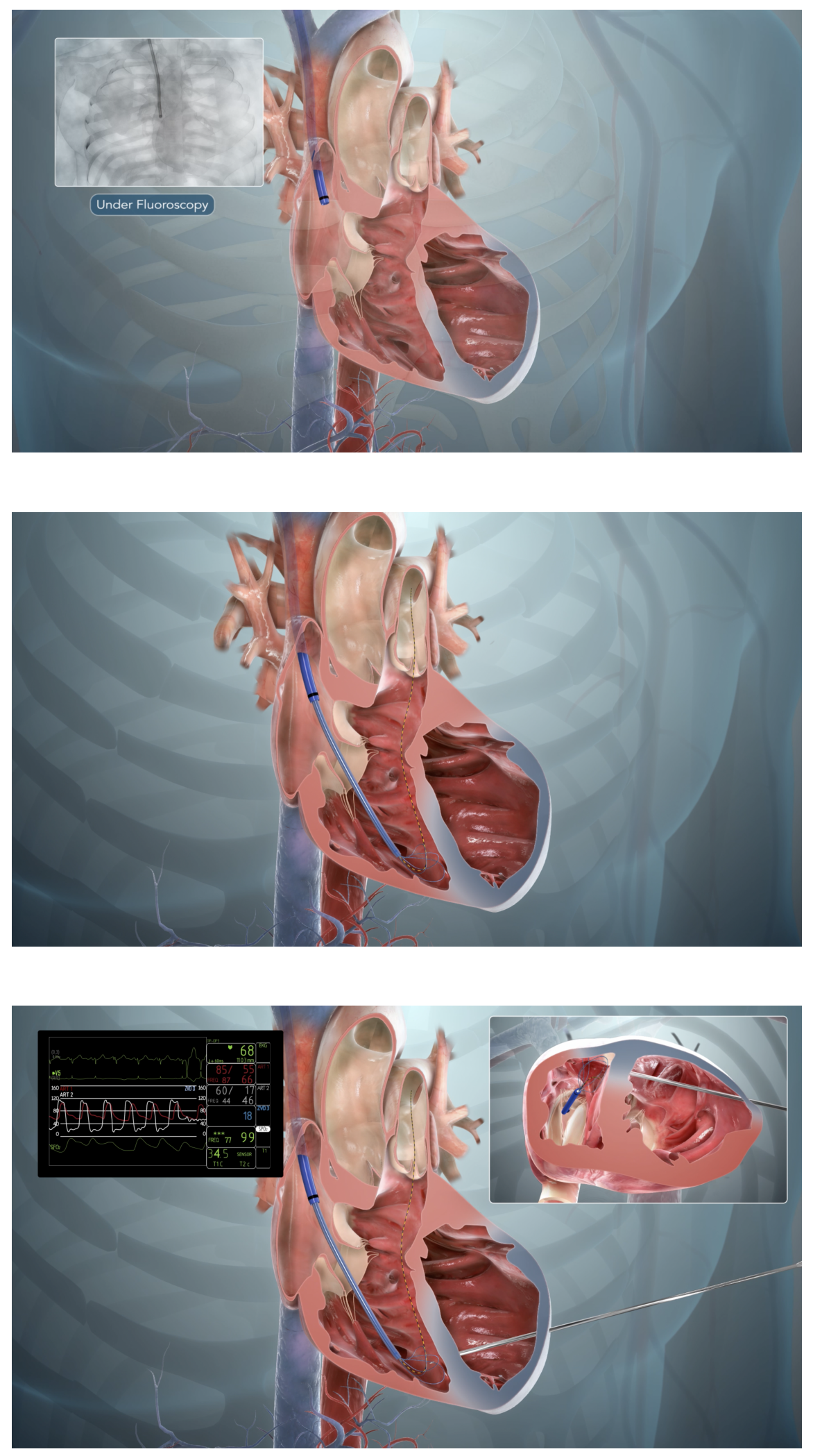

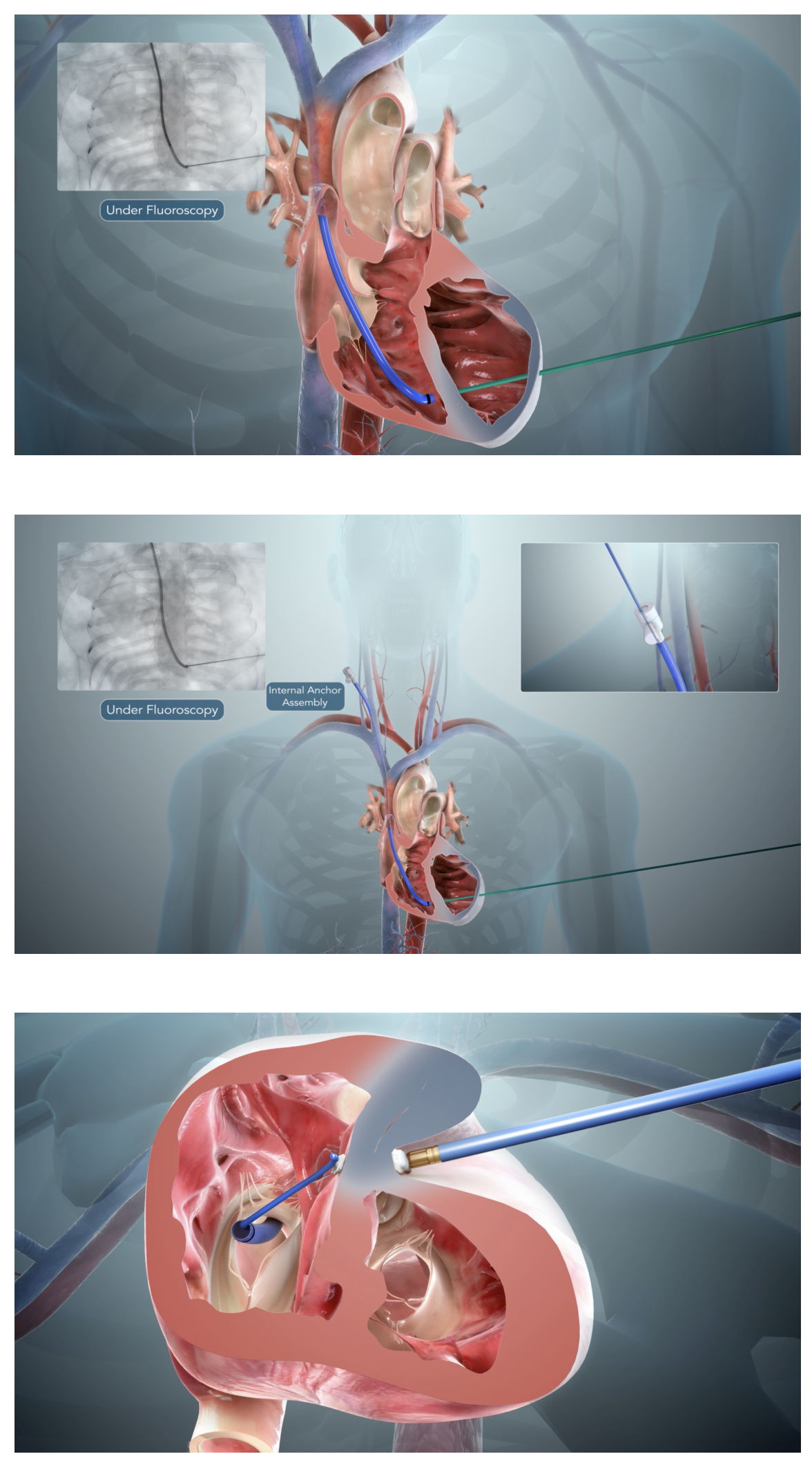


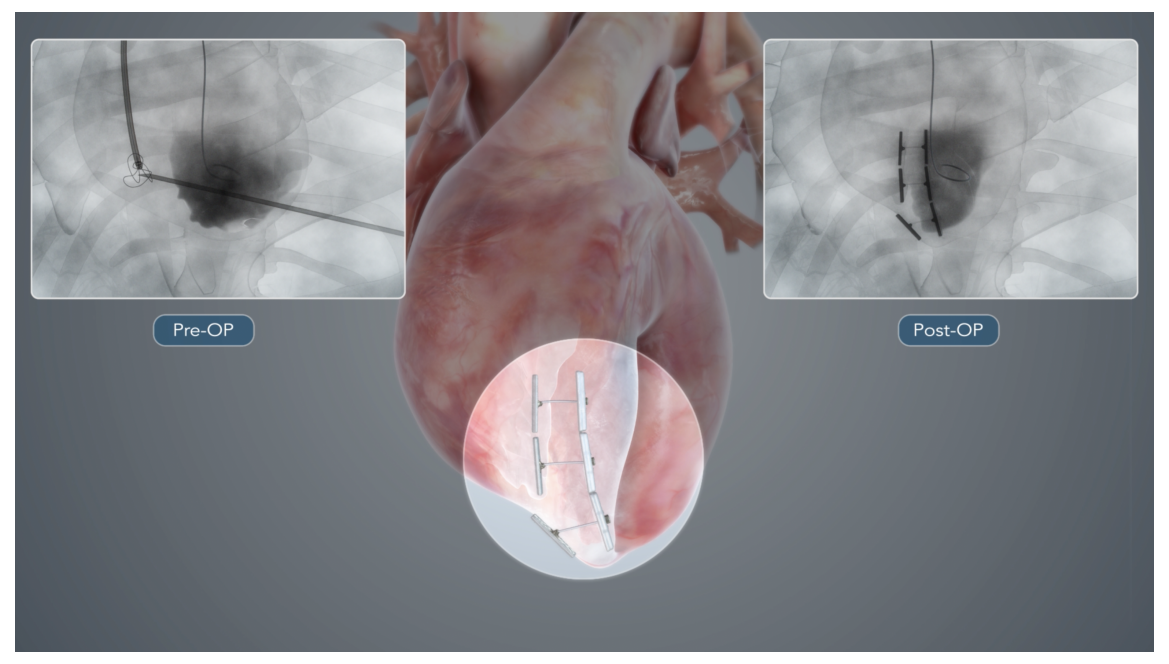

